\title{
Approach towards Car Failure Diagnosis-An Expert System
}

\author{
Yash Jindal \\ B.Tech (CS, $7^{\text {th }}$ Sem) \\ IPEC, UPTU \\ Ghaziabad, India \\ Rashi Aggarwal \\ B.Tech (CS, 7th Sem) \\ IPEC, UPTU \\ Ghaziabad, India
}

\author{
Swati Jain \\ B.tech (CS, 7th Sem) \\ IPEC, UPTU \\ Ghaziabad, India \\ Ms. Neeta Verma \\ A.P (CSE) \\ IPEC, UPTU \\ Ghaziabad, India
}

\begin{abstract}
The paper presents the processes that play a significant role in the development of an expert system. The research has been done to assist in the designing of an expert system for car failure diagnosis and repairs under constraint like time, place and availability of human expertise. Study of technologies for designing expert systems was undertaken to conclude best means, which are simple and easy, to implement and maintain while developing an expert system.
\end{abstract}

\section{Categories and Subject Descriptors}

[Computer Science/ Information Technology]: Artificial Intelligence -Expert system for car failure diagnosis.

\section{General Terms}

The paper deals with the Design of an expert system.

\section{Keywords}

Expert system, Knowledge acquisition, Inference process, Knowledge base, prolog.

\section{INTRODUCTION}

Expert System is a branch of artificial intelligence, which studies the field of developing and implementing computer programs that can act and work on the lines of human brain which can acquire knowledge and develop intelligence of its own to act accordingly in situations that may be entirely or partially new to it. It is a kind of intelligent computer program, that uses a knowledge base and inference engine to solve the problems solved only by experts. Expert System consists of inference engine, knowledge base, knowledge acquiring subsystem, human-machine interface and interpreter. The core of the expert system is inference engine and knowledge base. An inference engine is a computer program that tries to derive answers from a knowledge base. It is the "brain" that expert systems employ to reason about the information in the knowledge base for the ultimate purpose of formulating new conclusions. Knowledge base is used to store the knowledge of experts, when expert system is reasoning, it is to acquire knowledge and rules from the knowledge base, and repeat matching process of fault conditions to find an otherwise difficult solution for a given domain.

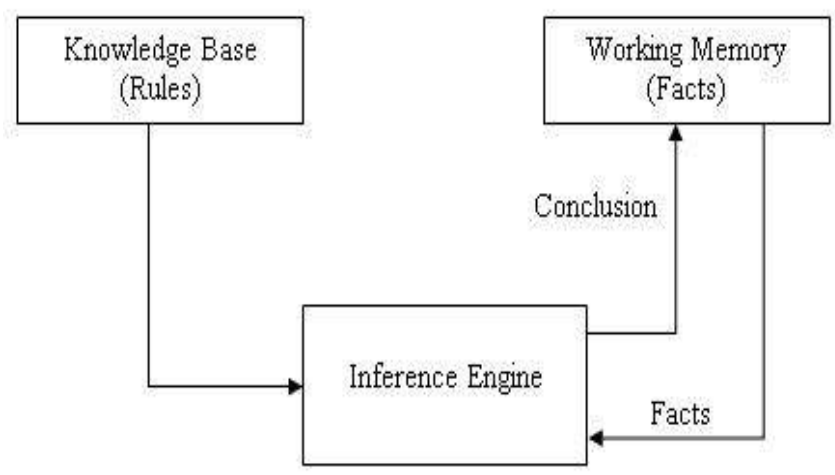

Figure1. Expert System's working design.

\subsection{Problem Definition}

A large segment of the car driving population is constituted by drivers who have little or very less information regarding troubleshooting a car, thus to assist such people in case of a breakdown where time, place and availability of human expert is a problem.

\subsection{Problem Review}

Expert System has been applied in many ways and various fields which are mean to make human's life simple and even easier. the most successful application of Artificial Intelligence (AI) in decision making so far is the development of Decision Support System (DSS), particularly expert system, which is a computer program that act as a 'consultant' or 'advisor' to decision makers. Expert systems are cheaper compared to human experts in the long-term scenario. However, expert systems are relatively costly to develop but easy and cheap to operate. An expert system attempts to emulate how a human expert solves a problem, mostly by the manipulation of symbols instead of numbers. Expert systems have been used in a wide variety of fields and have been highly successful in their specific areas of implementation as in the field of medicine. 


\subsection{Research Objective}

To develop an expert system for assisting in car failure diagnosis and troubleshooting while examining and identifying the stages, requirements, and criteria of designing an expert system.

\section{IDENTIFICATION OF COMPONENTS OF EXPERT SYSTEM}

An expert system is a set of programs that manipulate encoded knowledge to solve problems in a domain that normally requires human expertise. The expert system features given below which distinguishes it from conventional computer programs help identifying the components of an expert system.

- $\quad$ expert system uses knowledge rather than data.

- $\quad$ knowledge is encoded and maintained as separate entity from control program.

- It can explain how a conclusion was reached and why requested information is needed.

- Uses symbolic representation and reasons meta knowledge.

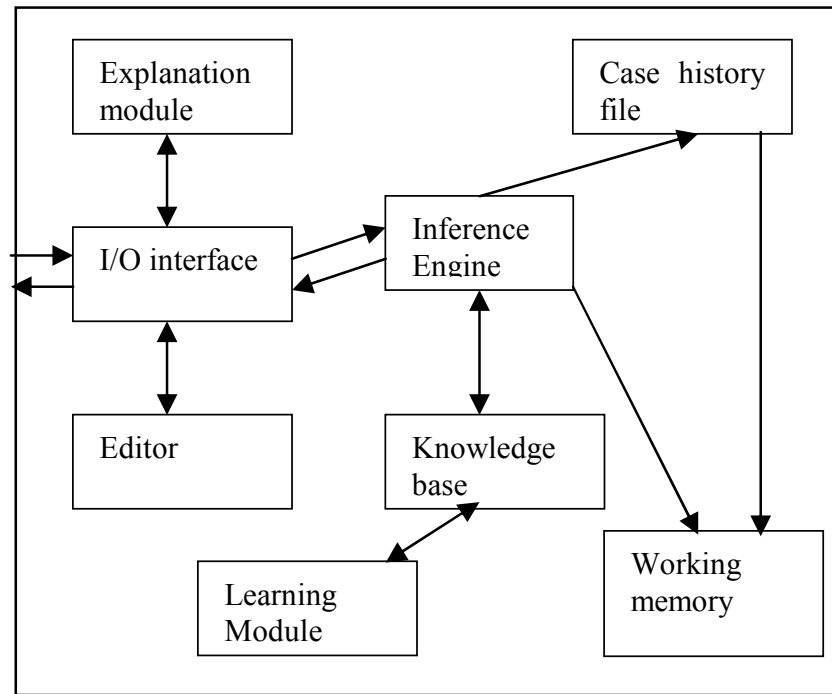

Figure4. Components of Expert system

The main components of an expert system thus are: i)The Knowledge Base ii) Editor iii) The Inference process iv) Learning Module v) Explanation Module vi) The I/O interface. The knowledge base is a collection of rules and facts that are used by the inference engine to obtain conclusions, editor is used to edit rules, learning module provides learning capability to system and history file maintains a history log from the working memory of the system.

\subsection{Knowledge Acquisition}

The critical part of knowledge acquisition is the process of collecting information from car experts, workshops, journals databases of problem queries, manuals of cars and from day to day learning of car problems from experiences and interaction with drivers naive as well as experts.

\begin{tabular}{|l|}
\hline $\begin{array}{l}\text { Domain } \\
\text { expert }\end{array}$ \\
\hline
\end{tabular}

Knowledge engineer

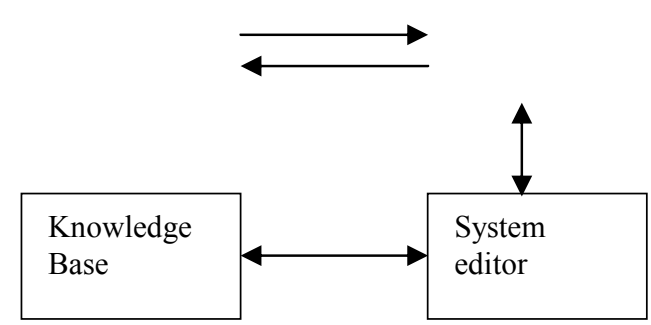

Figure3. Knowledge Acquisition

To elicit required knowledge, a knowledge engineer conducts extensive interviews with domain experts, during which typical problems and their solutions are evaluated, using the knowledge gained engineer codes the knowledge in form of rules. This knowledge is used to solve sample problems for review and validation by experts. The process is repeated sufficient number of times to acquire a sufficient database for problem domain.

\subsection{Inference Process}

The inference engine excepts user input queries and responses to question through the $\mathrm{i} / \mathrm{o}$ interface and uses dynamic knowledge together with static knowledge of the database to derive conclusions about situations. the process is recursive and carried in three stages as shown, 1)Match 2) select and 3)Execute

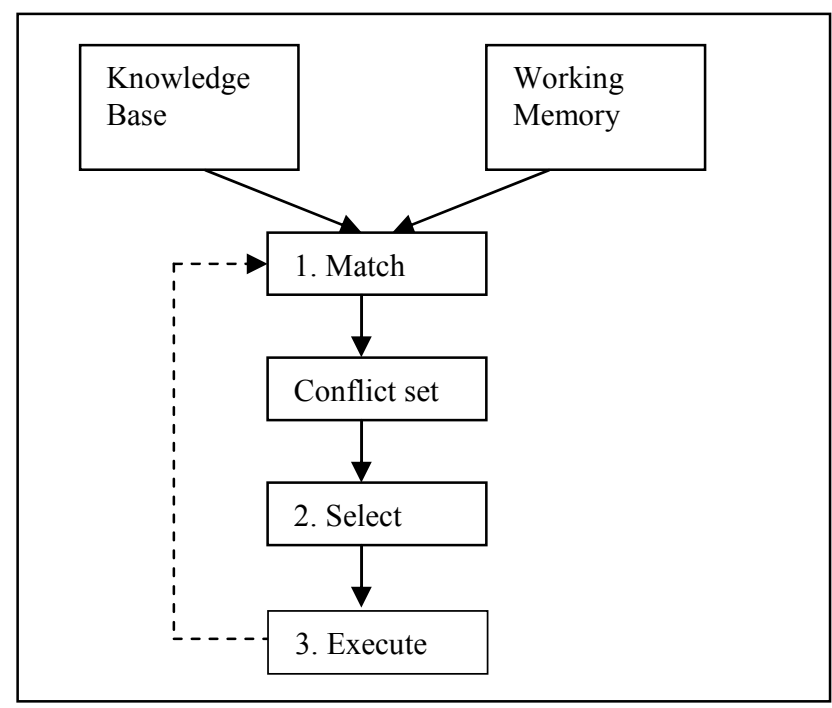

Figure4. The Inference process.

\subsection{Knowledge Base}

The Knowledge base is the set of rules created after a sufficient body of knowledge has been gathered about the subject domain. the car expert system requires all the knowledge for why a car wont' start represented in form of rules that can be answered in a yes-no format by the user. The study of languages for the purpose concluded prolog as a suitable language for representing the knowledge base for the following purposes:

PROLOG is one of the most unusual computer languages ever invented. It cannot be compared to FORTRAN, PASCAL, "C", or BASIC. The facilities complement, rather than replace those of conventional languages. Although it has great potential for database work, it has nothing in common with the database 
languages used on microcomputers Perhaps the best point to make is that while conventional languages are prescriptive, PROLOG is descriptive. For a car expert system a number of rules can be put together which are independent of each other and each relating to a specific fact, wherein the facts can be dependent on each other thus a man who is a specialist on car bodies can say his thing, the wheel specialist can have his say, and the participants can work with relative independence. And this brings to light a major advantage of PROLOG. Although PROLOG does not eliminate the need for task coordination, the problem is considerably simplified. Consider for example a simple set of rules for "why a car wont' start" represented in prolog:

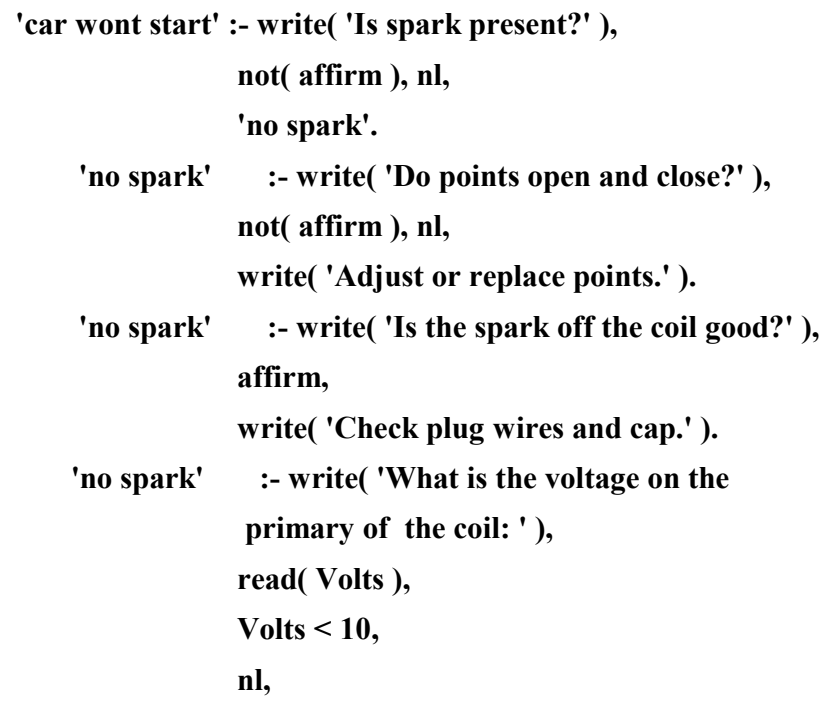

write('Check wiring and ballast resistor.').

\section{SYSTEM ANALYSIS AND DESIGN}

The analysis of the system developed shows that the knowledge acquired in the knowledge acquisition phase lead to the design of the system which includes the overall structure of the system's knowledge, the programming part and the interfaces. After considering all the components of an expert system an expert system prototype for car failure diagnosis was created by using rule based knowledge base defined in prolog, the process provided valuable insight into stages that lead to development of a successful expert system.

Designing phase was considered as a phase of the prototype development. In this project case, the final product is also in a way a prototype, in the design phase, the partial prototype was built which represented the initial part of the full prototype expert system. The partial prototype was built for the purpose of providing a deeper insight and better understanding of the problem and system's requirements. Thus the system's design is inherently an iterative process where findings from system testing are used to refine the system's knowledge and structure.

\section{CONCLUSION.}

Expert systems are gradually becoming a part of life and providing enhanced support to the already intelligent human race in its daily life, all because of their ability to act intelligently. In this case the expert system may be useful to execute the routine works and let the human expert to do the rest especially the more difficult jobs. The prototype of this system was developed in a limited time and resources and thus it is not that compatible and useful enough to be implemented in the real world yet. There must be so many other works to be taken in refining the errors and rules before it can really be used in the real situation. When this is done, the Expert System for Car failure diagnosis is ready to be used to assist all the car owners out there in situation where they are having problem with their cars and they can do it by themselves. Time and distance is no more a constraints to them.

\section{ACKNOWLEDGMENTS}

Our thanks to ACM SIGCHI for allowing us to modify templates they had developed. The research was supported by the computer science faculty and the director of Inderprastha Engineering College, U.P, India.

\section{REFERENCES}

[1] Fedra, K. and Winkelbauer, L., "A hybrid expert system, GIS and simulation modeling for environmental and technological risk management", Environmental Software \& Services GmbH, 2002.

[2] Jim Prentzas, et. al., "AWeb-Based ITS Controlled by a Hybrid Expert System”, Proceedings of IEEE International Conference on Advance Learning Techniques (ICALT'01), 2001.

[3] Markham H.C, "An internet-based expert system for teaching introductory data structures", Proceedings of the seventh annual consortium for computing in colleges central plains conference on The journal of computing in small colleges, pages $155-165,2001$.

[4] Rahman \& Bathnagar, "An Expert System Based Algorithm For Short Term Load Forecast", IEEE Transactions on Power Systems, Vol. 3, No. 2, May 1988.

[5] Nazar M. Zaki and Mohd Daud, "Development of a Computer-Aided System for Environmental Compliance Auditing", Journal of Theoretic, 2001. 